\title{
Plant cytochrome P450s: nomenclature and involvement in natural product biosynthesis
}

\begin{abstract}
Cytochrome P450s constitute the largest family of enzymatic proteins in plants acting on various endogenous and xenobiotic molecules. They are monooxygenases that insert one oxygen atom into inert hydrophobic molecules to make them more reactive and hydrosoluble. Besides for physiological functions, the extremely versatile cytochrome P450 biocatalysts are highly demanded in the fields of biotechnology, medicine, and phytoremediation. The nature of reactions catalyzed by P450s is irreversible, which makes these enzymes attractions in the evolution of plant metabolic pathways. P450s are prime targets in metabolic engineering approaches for improving plant defense against insects and pathogens and for production of secondary metabolites such as the anti-neoplastic drugs taxol or indole alkaloids. The emerging examples of P450 involvement in natural product synthesis in traditional medicinal plant species are becoming increasingly interesting, as they provide new alternatives to modern medicines. In view of the divergent roles of P450s, we review their classification and nomenclature, functions and evolution, role in biosynthesis of secondary metabolites, and use as tools in pharmacology.
\end{abstract}

Keyword: Medicinal plants; Pharmacology; Terpenoids; Plant-derived drugs 